\title{
Radiological differences between chronic thromboembolic pulmonary disease (CTEPD) and chronic thromboembolic pulmonary hypertension (CTEPH)
}

\author{
Carmine Capone $^{1,2}$ - Adele Valentini $^{3} \cdot$ Silvia Lina Spinillo ${ }^{1}$. Catherine Klersy ${ }^{4}$ - Anna Celentano ${ }^{1}$. Maurizio Pin ${ }^{1,5}$. \\ Cristian Monterosso ${ }^{6} \cdot$ Roberto Dore $^{7}$ - Emilio Maria Bassi ${ }^{3}$. Michela Zacchino ${ }^{3}$. Giuseppe Rodolico ${ }^{3}$. \\ Angelo Guido Corsico ${ }^{8,9} \cdot$ Lorenzo Preda $^{1,3} \cdot$ Stefano Ghio $^{10} \cdot$ Andrea Maria D'Armini ${ }^{1,11}$
}

Received: 21 June 2020 / Revised: 26 September 2020 / Accepted: 6 November 2020 / Published online: 28 January 2021

(C) The Author(s) 2021

\begin{abstract}
Objectives The aim of this study was to describe the radiological features of chronic thromboembolic pulmonary disease (CTEPD), not yet systematically described in the literature. Furthermore, we compared vascular scores between CTEPD and chronic thromboembolic pulmonary hypertension (CTEPH) patients, trying to explain why pulmonary hypertension does not develop at rest in CTEPD patients.

Methods Eighty-five patients (40 CTEPD, 45 CTEPH) referred to our centre for pulmonary endarterectomy underwent dualenergy computed tomography pulmonary angiography (DE-CTPA) with iodine perfusion maps; other 6 CTEPD patients underwent single-source CTPA. CT scans were reviewed independently by an experienced cardiothoracic radiologist and a radiology resident to evaluate scores of vascular obstruction, hypoperfusion and mosaic attenuation, signs of pulmonary hypertension and other CT features typical of CTEPH.

Results Vascular obstruction burden was similar in the two groups ( $p=0.073$ ), but CTEPD patients have a smaller extension of perfusion defects in the iodine map $(p=0.009)$ and a smaller number of these patients had mosaic attenuation $(p<0.001)$ than CTEPH patients, suggesting the absence of microvascular disease. Furthermore, as expected, the two groups were significantly different considering the indirect signs of pulmonary hypertension $(p<0.001)$.

Conclusions CTEPD and CTEPH patients have significantly different radiological characteristics, in terms of signs of pulmonary hypertension, mosaic attenuation and iodine map perfusion extension. Importantly, our results suggest that the absence of peripheral microvascular disease, even in presence of an important thrombotic burden, might be the reason for the absence of pulmonary hypertension in CTEPD.

Key Points

- CTEPD and CTEPH patients have significantly different radiological characteristics.

- The absence of peripheral microvascular disease might be the reason for the absence of pulmonary hypertension in CTEPD.
\end{abstract}

Andrea Maria D’Armini

andreamaria.darmini@unipv.it

1 Department of Clinical, Surgical, Pediatric and Diagnostic Sciences, Faculty of Medicine and Surgery, University of Pavia, Pavia, Italy

2 Diagnostic Imaging Service, ICS Maugeri, Via Salvatore Maugeri 10, 27100 Pavia, PV, Italy

3 Radiology Department, Fondazione IRCCS Policlinico San Matteo, Pavia, Italy

4 Clinic Epidemiology and Biometry Unit, Fondazione IRCCS Policlinico San Matteo, Pavia, Italy

5 Unit of Cardio-Thoracic Surgery, Maria Cecilia Hospital SpA, Cotignola, Emilia-Romagna, Italy
6 Division of Cardiac Surgery, Fondazione IRCCS Policlinico San Matteo, Pavia, Italy

7 Radiology Unit, Gruppo San Donato, Cinical Institute Citta di Pavia, Pavia, Italy

8 Respiratory Diseases Unit, Department of Medical Sciences and Infectious Diseases, ERN Lung, Fondazione IRCCS Policlinico San Matteo, Pavia, Italy

9 Department of Internal Medicine and Therapeutics, Faculty of Medicine and Surgery, University of Pavia, Pavia, Italy

10 Division of Cardiology, Fondazione IRCCS Policlinico San Matteo, Pavia, Italy

11 Cardiac Surgery and Pulmonary Hypertension Unit, Fondazione IRCCS Policlinico San Matteo, Pavia, Italy 
Keywords Pulmonary hypertension $\cdot$ Pulmonary embolism $\cdot$ Dual-energy computed tomography

\author{
Abbreviations \\ CT Computed tomography \\ CTEPD Chronic thromboembolic pulmonary disease \\ CTEPH Chronic thromboembolic pulmonary hypertension \\ CTPA Computed tomography pulmonary angiography \\ DSA Digital subtraction angiography \\ PEA Pulmonary endarterectomy \\ RHC Right heart catheterisation \\ TLCO Transfer factor of the lung for carbon monoxide
}

\section{Introduction}

Chronic thromboembolic pulmonary disease (CTEPD) is characterised by the presence of chronic thromboembolic material in the pulmonary arteries without pulmonary hypertension at rest. CTEPD patients represent a small proportion of the patients referred to expert centres, with symptoms and quality of life that may be as poor as those of patients with chronic thromboembolic pulmonary hypertension (CTEPH). Therefore, some of these patients are currently offered the same surgical or interventional treatment as patients with CTEPH $[1,2]$.

However, there are a number of gaps of evidence regarding this pathological condition.

It is yet unclear why pulmonary hypertension does not develop at rest in such patients; this could be either because the number of occluded segments is insufficient to affect resistance at rest or because no distal vasculopathy has developed as in CTEPH patients. In addition, it is unknown whether exercise limitation is due to exercise-induced pulmonary hypertension, developing in CTEPD due to an increased slope of the pulmonary arterial pressure-flow relationship, or to deadspace ventilation, with increased ventilatory equivalents for carbon dioxide $[3,4]$. The natural history of CTEPD is unknown and there is no evidence that CTEPD necessarily evolves to CTEPH [5].

Finally, a relevant gap of evidence in clinical practice is that the radiological features of CTEPD are not systematically described in the literature. Current belief is that angiography, whether computed tomography pulmonary angiography (CTPA) or digital subtraction angiography (DSA), shows in CTEPD patients the typical findings of CTEPH patients [5].

Accordingly, the aim of this study was to compare imaging scores of vascular obstruction and distal perfusion in patients with CTEPD and CTEPH [6-9]. We reasoned that, in the hypothesis that pulmonary hypertension does not develop in CTEPD because the number of occluded segments is insufficient to affect resistance at rest, imaging should demonstrate lesser degree of arterial obstruction in CTEPD. On the contrary, in the hypothesis that pulmonary hypertension does not develop in CTEPD because no distal vasculopathy has developed, imaging could demonstrate better indices of distal perfusion in CTEPD than in CTEPH patients.

\section{Materials and methods}

\section{Study subjects}

Between April 1994 and April 2019, a total number of 936 patients were referred to our centre and underwent pulmonary endarterectomy (PEA); out of these, we retrospectively evaluated all consecutive patients with CTEPD who underwent PEA ( $n=$ 46). A similar number of consecutive patients $(n=45)$ with CTEPH who underwent PEA in our centre between January 2014 and June 2016, having similar age and sex, was used as the control group, selected from a total number of 97 patients.

The only comorbidity which was considered as an exclusion criterion in both groups was the concomitant presence of parenchymal lung disease, on the basis of ventilation/ perfusion scintigraphy data and of the presence of advanced pulmonary emphysema and/or fibrosis at CT (30 patients were excluded only in the CTEPH group).

None of the patients enrolled in our study was referred to our centre for acute pulmonary embolism.

Patients were diagnosed as CTEPH or CTEPD on the basis of all examinations, including right heart catheterisation (RHC) at rest and imaging (CTPA and V/Q scintigraphy), consistently suggested by international guidelines over the study enrollment period [10].

Preoperatively, patients also underwent echocardiographic and respiratory function evaluations.

The respiratory evaluation included spirometry, singlebreath transfer factor of the lung for carbon monoxide (TLCO) and arterial blood gas analyses.

The study was approved by the Institutional Review Board of Fondazione IRCCS Policlinico San Matteo (protocol number 20200031903).

\section{Methods}

In CTEPD patients studied before $2009(n=6)$, examinations were performed on a 16-slice single-source CT scanner (Somatom Sensation, Siemens Healthineers); all other examinations (both in CTEPD and in CTEPH patients) were performed on a dual-source 64-slice CT scanner (Somatom Definition, Siemens Healthineers). 


\section{CT protocols}

In all patients, two consecutive angiographic scans were performed, in full inspiration.

- Scan parameters for 16-slice CT were as follows: $120 \mathrm{kV}$ with tube current $150 \mathrm{~mA}$; collimation $16 \times 0.75$; gantry rotation time $50 \mathrm{~ms}$; pitch 0.85 . Modulation of the milliamperage was routinely used (Care Dose 4D, Siemens Healthineers).

Pulmonary angiographic acquisition was made with bolus tracking technique, ROI (region of interest) on the main pulmonary artery (threshold $100 \mathrm{HU}$, scan delay $7 \mathrm{~s}$ ) and aortic angiographic acquisition with an 8-s delay, with caudo-cranial acquisition for both scans.

We used $100 \mathrm{ml}$ of contrast agent (iomeprol $400 \mathrm{mgI} / \mathrm{mL}$; Iomeron, Bracco), flush at $4 \mathrm{ml} / \mathrm{s}$, followed by $40 \mathrm{ml}$ of normal saline, flush at $4 \mathrm{ml} / \mathrm{s}$.

- Scan parameters for 64-slice dual-source CT were as follows: tube A, $140 \mathrm{kV}$ with tube current $60 \mathrm{~mA}$, FoV diameter $50 \mathrm{~cm}$; tube B, $80 \mathrm{kV}$ with tube current $300 \mathrm{~mA}$, FoV diameter $26 \mathrm{~cm}$; collimation $14 \times 1.2 \mathrm{~mm}$; gantry rotation time $30 \mathrm{~ms}$; pitch 0.7 . Modulation of the milliamperage was routinely used (Care Dose 4D, Siemens Healthineers).

Pulmonary angiographic acquisition was made with bolus tracking technique, ROI on the main pulmonary artery (threshold $100 \mathrm{HU}$, scan delay $4 \mathrm{~s}$ ), and aortic angiographic acquisition with a 4-s delay, with cranio-caudal acquisition for both scans.

We used $65 \mathrm{ml}$ of contrast agent (iomeprol $400 \mathrm{mgI} / \mathrm{mL}$; Iomeron, Bracco), flush at $5 \mathrm{ml} / \mathrm{s}$, followed by $40 \mathrm{ml}$ of normal saline, flush at $5 \mathrm{ml} / \mathrm{s}$.

\section{CTPA data reconstruction}

From the raw spiral projection data acquired with both tubes of the dual-source CT, "fused" lung and mediastinal images were generated by merging $60 \%$ of $140-\mathrm{kV}$ data with $40 \%$ of $80-\mathrm{kV}$ data via the process of linear blending [11-13], using medium-soft (D30) convolution kernel for mediastinal images and sharp (B80) convolution kernel for lung images, with 3$\mathrm{mm}$ and 1.5-mm thickness. Reconstructed images were transferred to a commercially available workstation (Leonardo, Siemens Healthineers) running Syngo software (Siemens Healthineers).

From both $80-\mathrm{kV}$ and $140-\mathrm{kV}$ images of the pulmonary angiographic scan, the software generated a dual-energy CT perfusion map, deriving the iodine content of each voxel using a three-material-decomposition algorithm for air, soft tissue and iodine [14]; colour-coded lung PBV images $7 \mathrm{~mm}$ in thickness were reconstructed at 14-mm intervals in both the axial and coronal planes using the same dual-energy application software.
From raw data of the single-source scanner, mediastinal and lung images were generated using very smooth (B10) convolution kernel for mediastinal images and ultra-sharp (B80) convolution kernel for lung images, with 1-mm thickness.

\section{Image analysis}

One cardiothoracic radiologist with more than 10 years of experience and a 5 th year radiology resident independently reviewed lung and pulmonary CTPA images and dualenergy CT perfusion maps in a blinded non-consecutive manner, using scores as described by Hoey et al [6].

Vascular obstruction Reviewing pulmonary CTPA images, a modified Qanadli index [15] as described by Hoey et al [6] was used to quantify the degree of arterial obstruction.

A maximal score of 2 was assigned for a patent segmental artery and its first-order subsegmental branches, giving a total possible score of 40 (three branches to both upper lobes, two branches to the middle lobe and lingula, and five branches to both lower lobes). One point was subtracted if there was clear evidence of first-order subsegmental occlusion, a segmental web stenosis or segmental partial occlusion. If two or more of these abnormalities were present, a score of 0 was assigned to that segment. Segmental total occlusion was also assigned a score of 0 . Laminated thrombus of the principal or lobar arteries that exceeded $50 \%$ of luminal reduction necessitated the subtraction of 1 from the scores for all segmental arterial branches distal to this point. Total vascular obstructive index was calculated by dividing the patient score by 40 and multiplying the result by 100 [15]. Scores for each lobe were expressed as a percentage of normal.

Mosaic hyperaemia Lung images were reviewed. In contrast to the original study [6], we decided to evaluate the ground glass alterations because more easily assessable, referred as "hyperaemic lung parenchyma".

We assessed the presence of hyperaemic lung parenchyma and the distribution of this alteration (central, peripheric or widespread). Then, a hyperaemic lung parenchyma score was derived by subjective assessment of each lobe for the extent (percentage score at 10\% increments; range, $0-100 \%$ ) of increased attenuation, which was defined as a subjective increase in parenchymal attenuation and containing vessels of larger than expected calibre, corresponding to areas of lung hyperaemia. A total "hyperaemia score" was calculated by applying a weighting factor to each lobar score and adding these together. The weighting factor was based on a 20 segment arterial anatomy model, as described by Qanadli et al [15]: the weighting factors were $3 / 20$ for each upper lobe, $2 / 20$ for the middle lobe and lingula, and 5/20 for each lower lobe. 
Dual-energy CT perfusion A dual-energy CT hypoperfusion score was derived by subjective assessment of the iodine map, generated from pulmonary angiographic scan. CTEPD patients who underwent $\mathrm{CT}$ on single-source scan were excluded from this evaluation.

Each lobe was scored according to the extent (percentage score at $10 \%$ increments; range, $0-100 \%$ ) of reduced perfusion (areas coloured dark red and black; areas of normal lung are coloured red). Map images were reviewed in axial, coronal and sagittal planes. For lobes not fully included in the dualenergy CT reconstruction field, in patients with thorax diameter exceeding the diameter of tube B FoV, a percentage was taken of the included portion of that lobe. Both readers used their experience to recognise and discount beam-hardening artifacts from undiluted contrast media and cardiac pulsation artifacts. To achieve a total hypoperfusion score, the individual scores for each lobe were weighted and added together in the same way as the mosaic attenuation score was derived.

We also evaluated the frequency of proximal pulmonary occlusion in both groups (considering thrombi obstructing $>50 \%$ of the lumen of a principal pulmonary artery).

Other parameters evaluated were the presence of CT indirect signs of pulmonary hypertension, in particular pulmonary artery (PA) diameter (maximum diameter on transversal images), the ratio between PA and aortic diameters (PA/aorta ratio), the ratio between right ventricle (RV) and left ventricle (LV) diameters ( $\mathrm{RV} / \mathrm{LV}$ ratio), the presence of $\mathrm{RV}$ hypertrophy (cut-off thickness $4 \mathrm{~mm}$ ) and interventricular septum bowing, the presence of systemic collateral arteries (bronchial, intercostal and phrenic arteries) and peripheral scars.

\section{Analysis}

Continuous data were described as median and interquartile range (IQR) and categorical data as counts and percent; data were compared with the Mann-Whitney $U$ test and the Fisher exact test, respectively. Bonferroni correction was applied for post hoc subgroup comparisons. The Spearman $R$ and its $95 \%$ confidence interval $(95 \% \mathrm{CI})$ were computed to assess the association of continuous variables. The Lin's concordance correlation coefficient and 95\% CI were calculated to measure the interobserver agreement in CT readings.

Stata 16 (StataCorp) was used for computations. A twosided $p$ value $<0.05$ was considered statistically significant.

\section{Results}

\section{Patients' characteristics (Table 1)}

The population of this study consisted of 91 patients ( 48 men and 43 women): 46 CTEPD ( 28 men and 18 women), 45 CTEPH (20 men and 25 women).

Table 1 Baseline characteristics

\begin{tabular}{|c|c|c|c|}
\hline & CTEPD group $(n=46)$ & CTEPH group $(n=45)$ & $p$ value \\
\hline \multicolumn{4}{|l|}{ Sex } \\
\hline M & $28(61 \%)$ & $20(44 \%)$ & $p=0.144$ \\
\hline Age & $61(43-71)$ & $64(53-69)$ & $p=0.769$ \\
\hline BMI & $27(25-30)$ & $26(23-28)$ & $p=0.088$ \\
\hline \multicolumn{4}{|l|}{ WHO functional class } \\
\hline III-IV & $21(46 \%)$ & $30(67 \%)$ & $p=0.058$ \\
\hline Disease duration (years) & $1.0(0.0-3.5)$ & $1.0(1.0-3.0)$ & $p=0.433$ \\
\hline PVR (WU) & $3.0(2.4-3.5)$ & $10.6(6.6-13.2)$ & $p<0.001$ \\
\hline mPAP $(\mathrm{mmHg})$ & $21(19-22)$ & $47(33-53)$ & $p<0.001$ \\
\hline CI & $2.6(2.3-3.0)$ & $2.0(1.7-2.5)$ & $p<0.001$ \\
\hline RA pressure $(\mathrm{mmHg})$ & $2(1-5)$ & $6(3-11)$ & $p<0.001$ \\
\hline $6 \mathrm{MWT}(\mathrm{m})$ & $430(317-490)$ & $297(228-378)$ & $p<0.001$ \\
\hline $\mathrm{PaO}_{2}(\mathrm{mmHg})$ & $79.8(70.5-86.8)$ & $68.3(60.9-76.7)$ & $p<0.001$ \\
\hline FEV1 (\% pred.) & $93(76-100)$ & $90(80-104)$ & $p=0.847$ \\
\hline FVC (\% pred.) & $96(82-102)$ & $100(88-113)$ & $p=0.100$ \\
\hline FEV1/FVC (\%) & $75(70-79)$ & $72(67-77)$ & $p=0.076$ \\
\hline TLCO (\% pred.) & $70(63-80)$ & $65(58-76)$ & $p=0.223$ \\
\hline
\end{tabular}

$B M I$ body mass index, $W H O$ World Health Organization, $P V R$ pulmonary vascular resistance, $W U$ Wood unit, $m P A P$ mean pulmonary artery pressure, $\mathrm{CI}$ cardiac index, $\mathrm{RA}$ right atrium, $6 \mathrm{MWT}$ 6-min walking test, $\mathrm{PaO}_{2}$ partial pressure of oxygen in arterial blood, $F E V 1$ forced expiratory volume in $1 \mathrm{~s} \%$ predicted, $F V C$ forced vital capacity $\%$ predicted, TLCO single-breath carbon monoxide transfer factor $\%$ predicted 
Table 2 CT characteristics

\begin{tabular}{lccc}
\hline & CTEPD group $(n=46)$ & CTEPH group $(n=45)$ & $p$ value \\
\hline Vascular obstruction score (\%) & $40(35-52.5)$ & $35(30-47.5)$ & $p=0.073$ \\
Mosaic hyperaemia & $8(17 \%)$ & $39(87 \%)$ & $p<0.001$ \\
Hyperaemia score (\%) & $20.25(9.8-26.3)$ & $20(14.5-27.5)$ & $p=0.581$ \\
Hypoperfusion score (\%) & $47(41-59)^{*}$ & $57(47-68)$ & $p=0.009$ \\
Proximal occlusion & $14(29 \%)$ & $5(11 \%)$ & $p=0.040$ \\
Peripheral scars & $44(92 \%)$ & $42(93 \%)$ & $p=1.000$ \\
Hypertrophied bronchial arteries & $44(94 \%)$ & $39(89 \%)$ & $p=0.476$ \\
Hypertrophied intercostal arteries & $28(60 \%)$ & $32(73 \%)$ & $p=0.268$ \\
Hypertrophied phrenic arteries & $25(53 \%)$ & $32(73 \%)$ & $p=0.082$ \\
RV hypertrophy & $15(31 \%)$ & $36(80 \%)$ & $p<0.001$ \\
Septum bowing & $2(4 \%)$ & $26(58 \%)$ & $p<0.001$ \\
RV/LV ratio & $0.99(0.86-1.1)$ & $1.3(1.1-1.61)$ & $p<0.001$ \\
PA diameter (mm) & $29(26-33)$ & $34(31-36)$ & $p<0.001$ \\
PA/aorta ratio & $0.91(0.84-1)$ & $1.09(1-1.16)$ & $p<0.001$ \\
\hline
\end{tabular}

$R V$ right ventricle, $L V$ left ventricle, $P A$ pulmonary artery

*Only 40 patients with CTEPD were evaluated for hypoperfusion score

As expected, patients with CTEPD had a less severe disease considering clinical conditions, right heart hemodynamic profile and functional capacity.

\section{CTPA findings (Table 2)}

- Vascular obstruction (Fig. 1) - median vascular obstructive score was $40 \%$ in the CTEPD group and $35 \%$ in the CTEPH group, with a non-significant difference between the two groups $(p=0.073)$.

- Proximal pulmonary obstruction-proximal obstruction was present in 14 patients (29\%) with CTEPD and in 5 patients $(11 \%)$ with CTEPH $(p=0.040)$.

- Hypoperfusion score (Fig. 2)-median hypoperfusion score was $47 \%$ in the CTEPD group and 57\% in the CTEPH group, with a significant difference between the two groups $(p=0.009)$.
- Lung hyperaemia (Fig. 3) - mosaic attenuation in lung parenchyma was observed in 8 patients $(17 \%)$ with CTEPD and in 39 patients $(87 \%)$ with CTEPH $(p<0.001)$; where present, there was no significant difference neither in its distribution nor in the hyperaemia score (median $20.25 \%$ in the CTEPD group, $20 \%$ in the CTEPH group; $p=0.581$ ).

- Pulmonary hypertension signs (Fig. 4) - a significant difference $(p<0.001)$ between the CTEPD and CTEPH groups was found for all indirect signs of pulmonary hypertension considered (RV hypertrophy, RV/LV diameters ratio, septum bowing, PA diameter and PA/aortic diameters ratio). Modest correlations were observed between PA diameter and both mPAP ( $r=0.472$; $p<0.001)$ and PVR $(r=0.477 ; p<0.001)$ and between PA/aorta ratio and both $\operatorname{mPAP}(r=0.429 ; p<0.001)$ and $\operatorname{PVR}(r=0.417 ; p<0.001)$.
Fig. 1 CT pulmonary angiography (coronal plane, MIP reconstructions). a CTEPD: proximal occlusion in the left interlobar pulmonary artery (black arrow) and bilateral segmental and subsegmental vascular obstructions. b CTEPH: bilateral segmental and subsegmental vascular obstructions
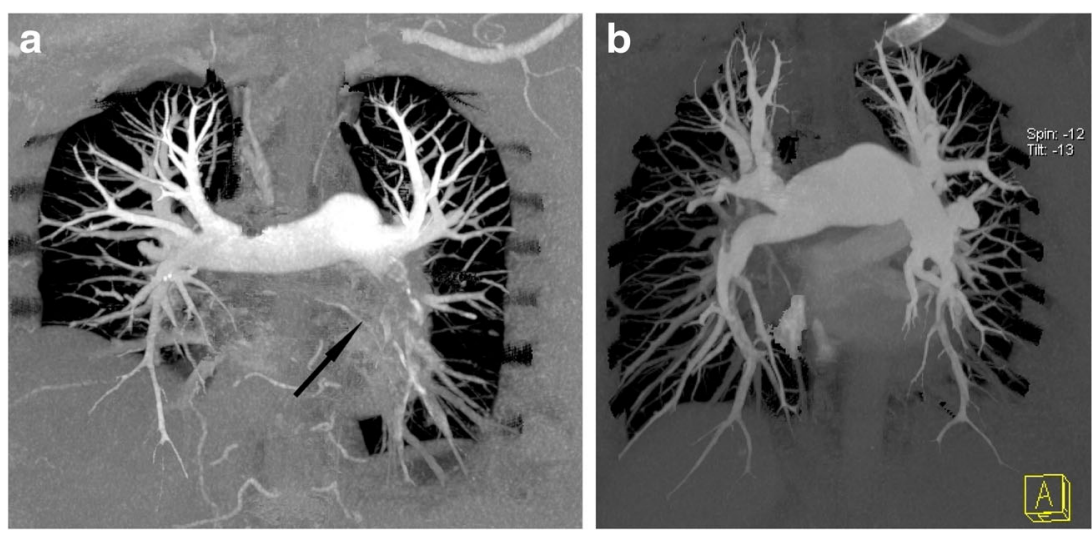
Fig. 2 Colour-coded CT iodine map shows a greater extent of perfusion defects (black areas) in CTEPH (b) rather than in CTEPD (a)
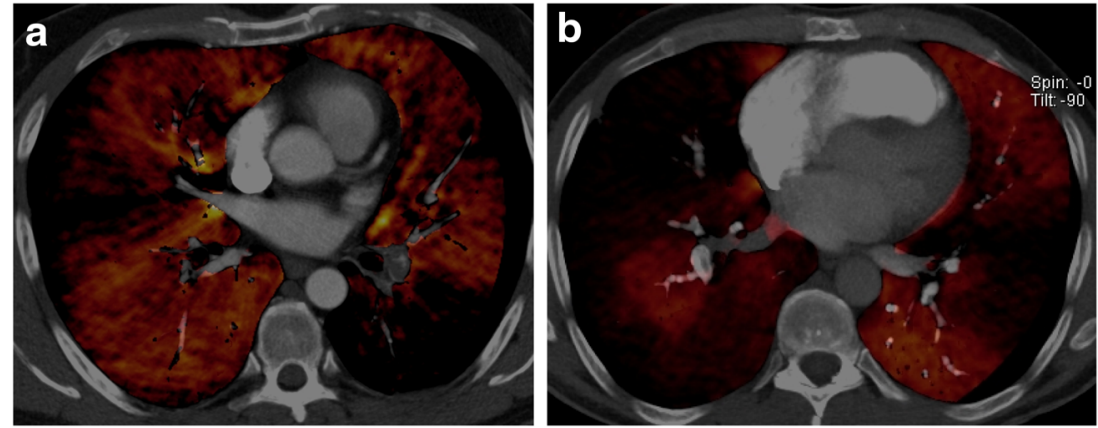

- Collateral systemic supply (Fig. 5) and peripheral scars (Fig. 3) -hypertrophic bronchial, intercostal and phrenic arteries were found in both CTED and CTEPH patients, without significant differences between the two groups ( $p$ $=0.476, p=0.268$ and $p=0.082$ respectively), such as peripheral scars $(p=1.000)$.

\section{Interobserver agreement}

Interobserver agreement was excellent for each of the CT scores evaluated.

The Lin's concordance correlation was very high for the vascular obstruction score $(r=0.92 ; 95 \%$ CI $0.87-0.97)$, the hypoperfusion score $(r=0.95 ; 95 \% \mathrm{CI} 0.92-0.98)$ and the hyperaemia score $(r=0.90 ; 95 \%$ CI $0.84-0.96)$.

\section{Discussion}

The main result of the present study is the identification of substantial differences in radiological findings of CTEPD and
CTEPH patients. Such differences can be clinically helpful to distinguish the two populations. Most importantly, these differences point at a different pathological substrate underlying CTEPD or CTEPH.

Patients with CTEPD showed a vascular obstruction burden which was similar to patients with CTEPH. As a matter of fact, proximal arterial obstruction (in particular of the right pulmonary artery) was more frequently observed in CTEPD patients than in the CTEPH population; to the best of our knowledge, this finding was never described before. Nevertheless, CTEPD patients had a lesser extension of perfusion defects in the iodine map. Peripheral vascular disease is not clearly visible in morphologic CTPA images, but it can be assessed as perfusion defects in iodine maps. In addition, mosaic hyperaemia, which is also an expression of microvascular peripheral disease, was found in a significantly smaller number of patients with CTEPD. Overall, these data strongly suggest that the absence of peripheral disease (i.e. of small vessel remodelling) might be the cause of the absence of pulmonary hypertension in CTEPD as compared to CTEPH patients.

No significant difference was found between the two groups considering the presence of collateral systemic supply; it is
Fig. 3 Lung window (b80 kernel reconstruction) CT. Lung hyperaemia is well evident in CTEPH (b) with both central and peripheral distribution, whereas is absent in CTEPD (a). Peripheral scars are present in both CTEPD (c) and CTEPH (d) patients
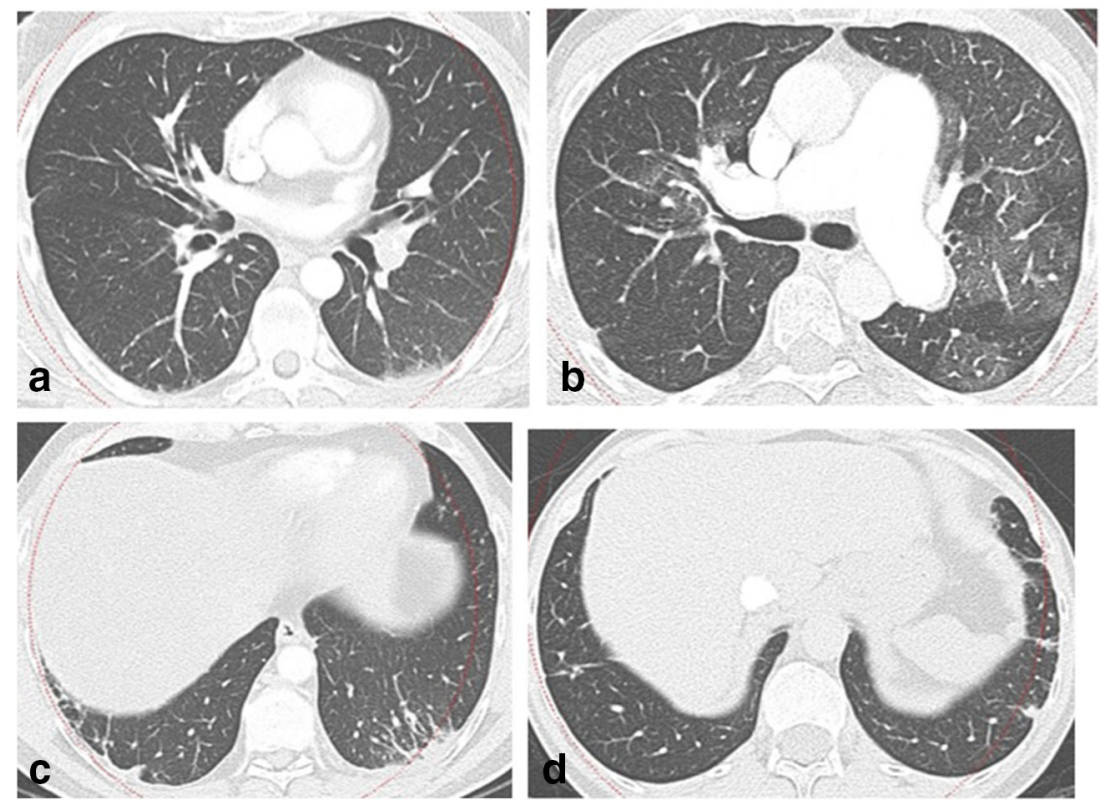
Fig. 4 CT signs of pulmonary hypertension. Normal heart diameters in CTEPD (a); in CTEPH patients (b) right ventricle dilation with septum bowing and heart leftward rotation are present. Important main pulmonary artery dilation in CTEPH (d), absent in CTEPD (c); proximal left main artery obstruction is evident in (c) (white arrow)
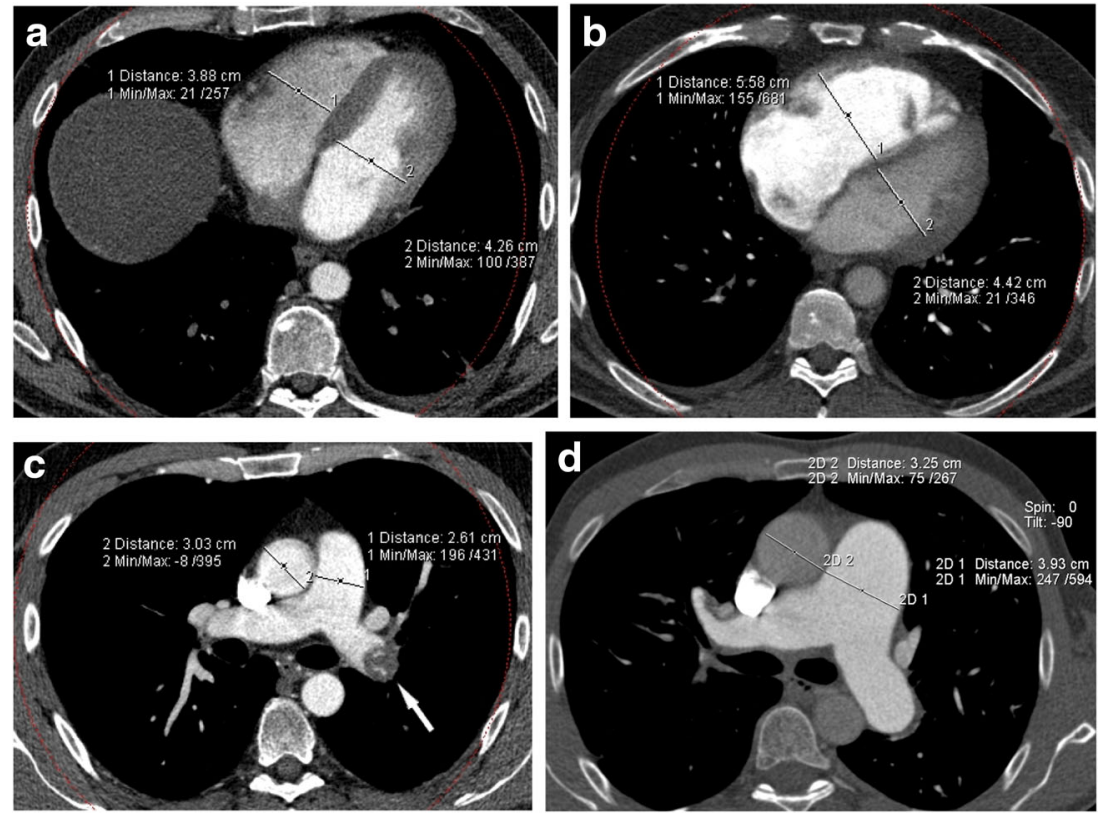

known that the presence of collateral systemic supply can help differentiating CTEPH from other forms of PH [16], but in our analysis, this finding was not useful to distinguish CTEPD from CTEPH patients. The hypothesis is that the development of collateral supply is a direct consequence of vascular obstruction, similarly present in both groups as a response to chronic lung ischemia, rather than a consequence of pulmonary hypertension.

Accordingly, no significant difference was found between the two groups in TLCO. In fact, TLCO is overestimated because of back-perfusion of the capillary bed by the extensive bronchial arterial collateral flow. This "luxury perfusion" plays a role in the maintenance of pulmonary parenchymal viability and in carbon monoxide exchange, although it does not improve the oxygen exchange $[17,18]$.

As expected, the two groups significantly differed substantially considering the indirect signs of pulmonary hypertension, such as an increased PA diameter and a PA/aorta diameter ratio $>1$ and $\mathrm{RV} / \mathrm{LV}$ ratio $>1$, which have already been described to correlate with the presence of pulmonary hypertension [19-22].

The main limitation of the present study is that the number of CTEPD patients evaluated is relatively small since it has been enrolled in a single centre and it is likely to represent a selected cohort of patients, as all these patients were referred for pulmonary endarterectomy being highly symptomatic. As a matter of fact, we lack population studies assessing the prevalence and the characteristics of symptomatic and asymptomatic patients with CTEPD and in which characteristics of such patients may differ from those who are referred to expert centres because of disabling symptoms.

We therefore acknowledge that validation in larger (and if possible unselected) series of patients is necessary, standardising the technical approach to the quantification of vascular obstruction and of lung perfusion.
Fig. 5 CT angiography (MIP reconstructions) shows hypertrophic bronchial arteries in both CTEPD (a) and CTEPH (b) patients (black arrows); a hypertrophic phrenic artery is also evident in (a) (white arrow)
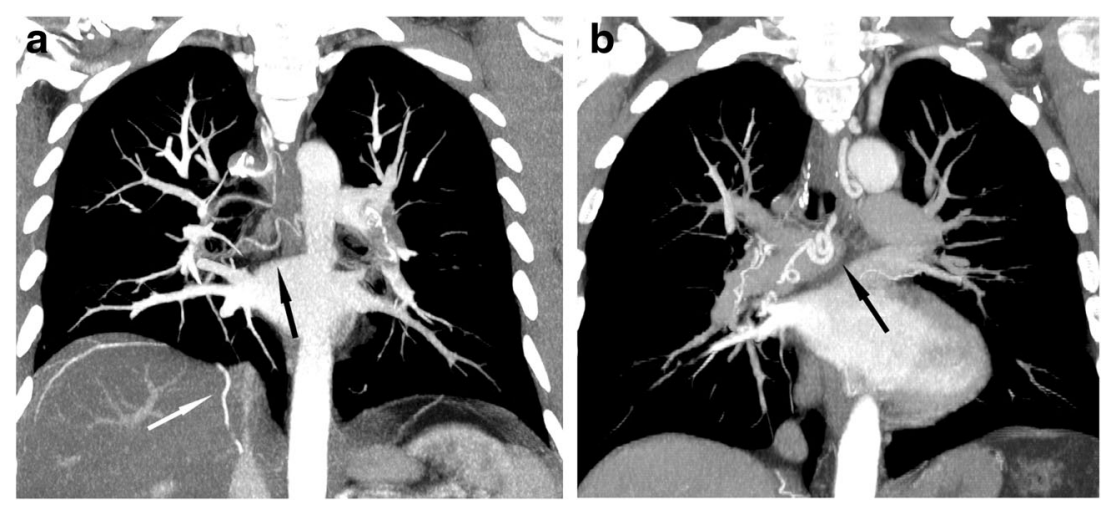
Another limitation is that CT protocols in CTEPD patients are not uniform, but the enrollment period lasted for several years since CTEPD is not a common condition and technical advances occurred during this period.

In conclusion, CTEPD patients show a vascular obstruction burden similar to CTEPH patients, but without CT signs of pulmonary hypertension and mosaic hyperaemia and with a smaller extension of perfusion defects in the iodine map; these findings could be useful to distinguish CTEPD from CTEPH patients. Eventually, identification of CTEPD population with DECT might in the future avoid performing an invasive procedure like right heart catheterisation.

Most importantly, these data suggest that the absence of peripheral microvascular disease, even in presence of an important thrombotic burden, might be the reason for the absence of pulmonary hypertension at rest in CTEPD.

Acknowledgements AV, SG and AMD had full access to all of the data in the study and take responsibility for the integrity of the data and the accuracy of the data analysis. CC, AV, SLS, CK, AC, MP, RD, AGC, SG and AMD contributed substantially to the study design, data analysis and interpretation, and the writing of the manuscript. All authors reviewed the final manuscript.

Funding Open access funding provided by Università degli Studi di Pavia within the CRUI-CARE Agreement.

\section{Compliance with ethical standards}

Guarantor The scientific guarantor of this publication is Stefano Ghio.

Conflict of interest The authors of this manuscript declare no relationships with any companies, whose products or services may be related to the subject matter of the article.

Statistics and biometry Catherine Klersy kindly provided statistical advice for this manuscript.

Informed consent Written informed consent was obtained from all subjects (patients) in this study.

Ethical approval Institutional Review Board approval was obtained.

\section{Methodology \\ - retrospective \\ - cross-sectional}

Open Access This article is licensed under a Creative Commons Attribution 4.0 International License, which permits use, sharing, adaptation, distribution and reproduction in any medium or format, as long as you give appropriate credit to the original author(s) and the source, provide a link to the Creative Commons licence, and indicate if changes were made. The images or other third party material in this article are included in the article's Creative Commons licence, unless indicated otherwise in a credit line to the material. If material is not included in the article's Creative Commons licence and your intended use is not permitted by statutory regulation or exceeds the permitted use, you will need to obtain permission directly from the copyright holder. To view a copy of this licence, visit http://creativecommons.org/licenses/by/4.0/.

\section{References}

1. Wiedenroth GB, Olsson KM, Guth S et al (2018) Balloon pulmonary angioplasty for inoperable patients with chronic thromboembolic disease. Pulm Circ 8(1):2045893217753122. https://doi.org/ $10.1177 / 2045893217753122$

2. Taboada D, Pepke-Zaba J, Jenkins D et al (2014) Outcome of endarterectomy in symptomatic chronic thromboembolic disease. Eur Respir J 44:1635-1645

3. van Kan C, van der Plas MN, Reesink HJ et al (2016) Hemodynamic and ventilatory responses during exercise in chronic thromboembolic disease. J Thorac Cardiovasc Surg 152:763-771

4. Held M, Kolb P, Grün M et al (2016) Functional characterization of patients with chronic thromboembolic disease. Respiration 91:503-509

5. Kim NH, Delcroix M, Jais X et al (2019) Chronic thromboembolic pulmonary hypertension (WSPH). Eur Respir J 53:1801915

6. Hoey ET, Mirsadraee S, Pepke-Zaba J, Jenkins DP, Gopalan D, Screaton NJ (2011) Dual-energy CT angiography for assessment of regional pulmonary perfusion in patients with chronic thromboembolic pulmonary hypertension: initial experience. AJR Am J Roentgenol 196(3):524-532

7. Liu M, Ma Z, Guo X, Zhang H, Yang Y, Wang C (2011) Computed tomographic pulmonary angiography in the assessment of severity of chronic thromboembolic pulmonary hypertension and right ventricular dysfunction. Eur J Radiol 80(3):e462-e469

8. Liu M, Ma Z, Guo X, Chen X, Yang Y, Wang C (2013) Cardiovascular parameters of computed tomographic pulmonary angiography to assess pulmonary vascular resistance in patients with chronic thromboembolic pulmonary hypertension. Int $\mathrm{J}$ Cardiol 164(3):295-300

9. Takagi H, Ota H, Sugimura K et al (2016) Dual-energy CT to estimate clinical severity of chronic thromboembolic pulmonary hypertension: comparison with invasive right heart catheterization. Eur J Radiol 85:1574-1580

10. Galiè N, Humbert M, Vachiery JL et al (2016) 2015 ESC/ERS guidelines for the diagnosis and treatment of pulmonary hypertension: the Joint Task Force for the Diagnosis and Treatment of Pulmonary Hypertension of the European Society of Cardiology (ESC) and the European Respiratory Society (ERS): endorsed by: Association for European Paediatric and Congenital Cardiology (AEPC), International Society for Heart and Lung Transplantation (ISHLT). Eur Heart J 37(1):67-119

11. Remy-Jardin M, Faivre JB, Pontana F et al (2010) Thoracic applications of dual energy. Radiol Clin North Am 48:193-205

12. Pontana F, Faivre JB, Remy-Jardin M et al (2008) Lung perfusion with dual-energy multidetector-row CT (MDCT): feasibility for the evaluation of acute pulmonary embolism in 117 consecutive patients. Acad Radiol 15:1494-1504

13. Zhang LJ, Zhao YE, Wu SY et al (2009) Pulmonary embolism detection with dual-energy CT: experimental study of dual-source CT in rabbits. Radiology 252:61-70

14. Johnson TR, Krauss B, Sedlmair M et al (2007) Material differentiation by dual energy CT: initial experience. Eur Radiol 17:1510-1517

15. Qanadli SD, El Hajjam M, Vieillard-Baron A et al (2001) New CT index to quantify arterial obstruction in pulmonary embolism: comparison with angiographic index and echocardiography. AJR Am J Roentgenol 176:1415-1420

16. Remy-Jardin M, Duhamel A, Deken V, Bouaziz N, Dumont P, Remy J (2005) Systemic collateral supply in patients with chronic thromboembolic and primary pulmonary hypertension: assessment with multi-detector row helical CT angiography. Radiology 235(1):274-281

17. Corsico AG, D'Armini AM, Cerveri I et al (2008) Long-term outcome after pulmonary endarterectomy. Am J Respir Crit Care Med 178:419424 
18. Hodson J, Graham A, Hughes JM, Gibbs JS, Jackson JE (2006) Pulmonary artery-to-pulmonary artery anastomoses: angiographic demonstration in patients with chronic thromboembolic pulmonary hypertension. Clin Radiol 61:259-263

19. Corson N, Armato SG, Labby ZE, Straus C, Starkey A, GombergMaitland M (2014) CT based pulmonary artery measurements for the assessment of pulmonary hypertension. Acad Radiol 21(4): $523-530$

20. Chan AL, Juarez MM, Shelton DK et al (2011) Novel computed tomographic chest metrics to detect pulmonary hypertension. BMC Med Imaging 11(1):7
21. Mahammedi A, Oshmyansky A, Hassoun PM, Thiemann DR, Siegelman SS (2013) Pulmonary artery measurements in pulmonary hypertension: the role of computed tomography. J Thorac Imaging 28(2):96-103

22. Edwards PD, Bull RK, Coulden R (1998) CT measurement of main pulmonary artery diameter. Br J Radiol 71(850):1018-1020

Publisher's note Springer Nature remains neutral with regard to jurisdictional claims in published maps and institutional affiliations. 